\title{
The Experience of Pay for Performance in English Family Practice: A Qualitative Study
}

Stephen M. Campbell, PbD

Ruth McDonald, PbD

Helen Lester, MD

National Primary Care Research and Development Centre, the University of Manchester, Manchester, UK

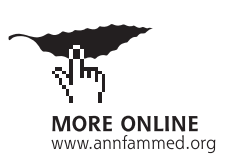

Conflict of interest: Helen Lester currently provides academic advice on evidence-based medicine to the national Quality and Outcomes Framework negotiating teams.

\section{CORRESPONDING AUTHOR}

Stephen M. Campbell, PhD National Primary Care Research and Development Centre

University of Manchester Williamson Bldg, Oxford Rd Manchester, M13 9PL, UK stephen.campbell@manchester.ac.uk

\begin{abstract}
PURPOSE We conducted an in-depth exploration of family physicians' and nurses' beliefs and concerns about changes to the family health care service as a result of the new pay-for-performance scheme in the United Kingdom (Quality and Outcomes Framework [QOF]).
\end{abstract}

METHODS Using a semistructured interview format, we interviewed 21 family doctors and 20 nurses in 22 nationally representative practices across England between February and August 2007.

RESULTS Participants believed the financial incentives had been sufficient to change behavior and to achieve targets. The findings suggest that it is not necessary to align targets to professional priorities and values to obtain behavior change, although doing so enhances enthusiasm and understanding. Participants agreed that the aims of the pay-for-performance scheme had been met in terms of improvements in disease-specific processes of patient care and physician income, as well as improved data capture. It also led to unintended effects, such as the emergence of a dual QOF-patient agenda within consultations, potential deskilling of doctors as a result of the enhanced role for nurses in managing long-term conditions, a decline in personal/relational continuity of care between doctors and patients, resentment by team members not benefiting financially from payments, and concerns about an ongoing culture of performance monitoring in the United Kingdom.

CONCLUSIONS The QOF scheme may have achieved its declared objectives of improving disease-specific processes of patient care through the achievement of clinical and organizational targets and increased physician income, but our findings suggest that it has changed the dynamic between doctors and nurses and the nature of the practitioner-patient consultation.

Ann Fam Med 2008;6:228-234. DOI: 10.1370/afm.844.

\section{INTRODUCTION}

I n April 2004 the United Kingdom government introduced a new General Medical Services Contract incorporating a pay-for-performance scheme (the Quality and Outcomes Framework, or QOF) for family doctors. The scheme attached financial rewards for meeting 146 quality targets in relation to clinical, organizational, and patient experience indicators, ${ }^{1}$ and in 2006 it was modified to 135 indicators. ${ }^{2}$ The intended consequences of the new contractual arrangements were to reward quality of care rather than numbers of registered patients, to improve data capture and care processes, and to improve patient outcomes and doctors' working conditions. ${ }^{1}$

The potential unintended consequences of such schemes are argued to include deleterious effects on clinician behavior and service provision. For example, the new scheme might have a negative effect on continuity of care and result in care fragmentation and a less holistic approach to patient care by doctors, as well as reduced quality for nonincentivized conditions and damage to doctors' professional motivation. ${ }^{1,3}$ 
The impact of pay-for-performance schemes is also likely to be influenced by a number of other factors. ${ }^{4-11}$ Such factors include levels of physician understanding of the purpose and involvement in the development of the scheme; the nature, appropriateness, and adequacy of measures used; the feasibility of implementation; and the magnitude of an incentive necessary to produce the behavioral change required to achieve targets. Other factors include the balance between team and individual incentives and the extent to which behavioral changes required to achieve targets are aligned to physician priorities and values.

Most research into the impact of the QOF has used quantitative methods and focused on associations between target achievement and, for example, practice characteristics, ${ }^{12-15}$ population characteristics, ${ }^{16}$ or the effect on quality for individual conditions, such as diabetes. ${ }^{17}$ There have been higher than expected achievement rates $^{14}$ and a modest accelerated improvement in patient-level outcomes with some conditions. ${ }^{18}$ QOF has cost the UK economy approximately $£ 1$ billion each year in payments to practices.

In contrast with most published work in this area, this study used a qualitative methodology. Although pay-for-performance schemes are being implemented in many countries, little is known about the views of health care clinicians of the intended and unintended effects of the incentives on their behavior, motivation, and daily working life. We describe an in depth-exploration of family physicians' and nurses' ideas, beliefs, and concerns about changes to the family health service in the United Kingdom.

\section{METHODS}

\section{Participants}

We carried out semistructured interviews with family doctors and nurses in practices across England between February and August 2007. The practices were drawn from a nationally representative cohort of practices, ${ }^{18,19}$ based on number of doctors working in the practice and the socioeconomic deprivation of the locality. The topic guide (Supplemental Appendix, org/cgi/content/full/6/3/228/DC1) was developed from the team's a priori questions and issues identified in previous work with the same practices undertaken by the study team. ${ }^{1,3}$

\section{Data Analyses}

We digitally recorded and fully transcribed all interviews. Data collection and analysis were concurrent and continued until the study team believed that theoretical saturation was complete. All transcripts were read by at least 2 of the research team independently, and a preliminary coding frame was constructed. We used a constant comparative method to interpret the data ${ }^{20}$ identifying key concepts using an open-coding method. Once coding was completed, the codes that had common elements were merged to form categories. All authors were involved in this process, and disagreements were discussed until a consensus was achieved. We actively sought disconfirming evidence and deviant case analyses throughout. ${ }^{21}$ Data analysis also took into account the different professional backgrounds of the participants. We decided early on in the data collection process not to use qualitative software, because all authors were responsible for collecting and analyzing the entire data set, and we felt sufficiently immersed in the data to code and interpret appropriately. All transcripts were kept intact to interpret responses in the context of the practice. All quotations have been chosen on grounds of representativeness.

One author (H.L.) is a primary care physician, and 2 (S.C., R.M.) are nonclinical health services researchers. All authors have a research interest in the quality of care within family medicine. The study had full ethical committee and research governance approval.

\section{RESULTS}

We invited 43 health professionals in the 22 practices to participate (22 physicians and 21 nurses). In 1 practice the practice nurse was on long-term sick leave. Forty-one of these family practice professionals (95\%) agreed to be interviewed ( 21 physicians and 20 nurses). Five of the physicians were female (aged between 46 and 64 years), and 16 were male (aged between 40 and 64 years). Only 1 physician worked part-time. All nurses were female; 17 worked part-time and 3 worked full-time (aged between 35 and 60 years). Overall, 169,000 patients were registered with the 42 practices, and practice sizes ranged from 1,773 to 18,700 registered patients. The 42 practices employed 110 physicians and 71 nurses. Physicians had also been interviewed in 22 of the same practices in 2004, with 60\% of doctors taking part in both years. ${ }^{3}$ In this article, we report the 4 major themes identified: the effects of implementing a pay-for-performance scheme; consultation process and agendas; performance monitoring and competition; and new indicators introduced in to the pay-for-performance scheme in 2006.

\section{Effects of Implementing}

\section{a Pay-for-Performance Scheme}

Most physicians believed that the quality targets had improved patient care by focusing attention on necessary clinical activities that might have been being 
neglected. Moreover, the targets had prompted staff to contact patients who were reluctant to attend, encouraged preventive work and chronic disease management, and promoted a sense of pride in achievement.

Most doctors and nurses acknowledged that nurses had become the primary provider of health care for patients with chronic conditions.

It is more likely that nurses could manage without family physicians than we could manage without nurses (General practitioner $[\mathrm{GP}] 8)$.

Although most doctors welcomed the added value of this role, some doctors believed they had become deskilled in areas previously seen as a core part of their role.

There's a little bit of deskilling there. I mean, we have a respiratory nurse, and so she sees all the asthmatics and does all the routine checks on them.... But a lot of the asthmatics tend not (to see a GP) which is a good thing, because it means they'll be better controlled. But on the other hand, I feel like, "Oh, an asthmatic patient! What do I do?" you know. "Oh, I'll send them to X," you know. It's like, that was a thing that I would have dealt with on my own before. But now it is better (GP10).

All interviewees recognized that QOF had led to considerable extra income at the practice level. Although the new contract is a practice contract, and targets had been achieved by teams of doctors, nurses, and administrative staff, most of additional money became part of the doctors' income as employers and owners of practices, regardless of the nurses' contribution to achieving the targets. One doctor described this additional money as like "having 5 year's pay rise in one go." Some doctors believed they had already been working to a high standard, and the extra money was an acknowledgment of this previously unpaid work.

And I think practices that were doing well anyway are still doing stuff above and beyond what's in the new contract, as they always have, and are perhaps now getting rewarded for work that they were doing (GP4).

The financial reward in return for extra work was also seen as helpful in raising morale within the profession and improving physician work-life balance. A minority of doctors, however, expressed concern about losing future control of patient care to the government because of, or perceived negative public opinion generated by, the increased family doctor income.

Well it's certainly improved my income. Probably increased my workload, not to the same degree as it increased my income. But I'm a bit worried that we've sold our soul to the devil to some degree, because they can change the goal posts later (GP39).
I don't think the $£ 250,000$ (media) headlines help much. I had an interesting discussion last week with a young man, a man my age - a 36-year-old guy-who was having a hard time of it at work, and he was talking about stress, and I said, "Oh yes, tell me about it," and he said, "Och, stress, look at this, this is a cushy number you've got ... you are getting plenty of money for it" (GP49).

That only a small financial bonus had been paid to nursing staff in some (but not all) practices created a sense of resentment among a considerable minority of the nurses. These nurses perceived their increased autonomy, hard work, and chronic disease management roles as critical in achieving QOF targets.

I'm sure the doctors appreciate what the nurses do, but I'm sure that we haven't had our salary updated as much as we should, for the money that they're probably getting from QOF. And that's, all 3 of us agree that. All the 3 nurses, we agree that we're doing a lot more of their work for them, and not much in the way of money recognition, yeah (Practice nurse $[\mathrm{PN}] 47$ ).

Continuity of care was claimed as a central feature of both doctor and practice nurse roles. For most nurses, interpersonal continuity was described as a relatively new feature as they assumed responsibility for patients with chronic conditions. Doctors were far more likely to stress the importance of their longitudinal relationship with patients, but some also stressed that the contract had helped to accelerate a preexisting decline in interpersonal continuity of care.

In the sense that it's still a patient presenting to a doctor with a problem, yes, it is the same as it always was. The difference is that it's more likely that the patient and the doctor won't know each other (GP25).

... with the asthma, the patients are beginning to see the same nurse, you know, rather than a different GP.... I will see the diabetics, and they know that I've been trying to say to them, "can you come, you know you can always come back," and I always try and make it so that there is open access for them if they have got a problem (PN49).

\section{Consultation Process and Agendas}

All doctors reported that there had been no change to the essence of the face-to-face doctor-patient interaction within routine consultations, which are booked at 10 -minute intervals. Addressing a number of agendas within a single consultation was seen by many doctors as a key skill of good family practice and reflected a long-standing acknowledgment that a consultation could include acute and chronic problems, health promotion, and health prevention advice as appropriate to that consultation. 
In some respects my role hasn't changed and never will do, as far as I can see, and not in my lifetime anyway. A person comes in the door, sits down and I ask them what's wrong and you try and fix it. That hasn't changed (GP21).

The context and consequences of the doctorpatient interaction, however, were perceived by all doctors as having changed as a direct result of the pay-for-performance scheme. All participants acknowledged that the QOF had influenced their agenda. Although not raised directly by interviewers, many participants used the concept and the term agenda spontaneously when describing the consultation.

... so it's made the 2 agendas a little bit clearer, and I guess you've always had a health agenda, which, I said before, if you come with a cold, your agenda and mine is probably never been the same, but now that mine is encapsulated by QOF ... it's a bit more blatantly not the same. So I think there is an intrusion there, and it's not an entirely patient-led agenda, because you've got things that you want to do that you think are more important (GP2).

So there is all this going on that he, the patient's agenda is one thing and yours is another ... your brain's working twice isn't it, at the same time (GP38).

The use of computerized chronic disease templates in consultations had been part of primary care in the United Kingdom for some years. Although many doctors and nurses believed the templates played a positive role in prompting them to take action, such as measuring blood pressure, others believed the templates introduced a new element into the consultation and threatened to shift the balance away from the patient's immediate agenda. Some doctors commented on how pursuing QOF targets within a consultation could create a potentially conflicting doctor and patient dual agenda. Others described incorporating the QOF agenda while attempting to deal with the patient's agenda, despite having reservations about the value of QOF work, whereas still others stated that this double agenda added to the workload of consultations and risked doctors being distracted from the patient's agenda.

... in terms of asking them additional questions to fit with QOF information that is required, I think that has altered, yes ... at times I feel uncomfortable with consultations where I have to include certain things related to that that I might not normally have deemed relevant at that particular point in time (GP52).

And there have been 1 or 2 occasions where I went through the cholesterol, the depression, the CHD, and everything else, and "Oh, that's wonderful, I'm finished now," and the patient said "Well, what about my foot then?" "What foot" (GP39)?

Some doctors and nurses stated they had made a point of apologizing or explaining to a patient why they were focusing on the targets as well as the patient's issues.

I feel actually I'm looking at the patient less than I used to, which is a shame... I have to say to them, "I'm sorry, I've got to look at the computer as well and type in while you're talking to me" (PN38).

All were keen, however, to emphasize that the patient's agenda came first and that QOF reminders flashed up on the computer would be bypassed if there was insufficient time to address both. Most doctors also stated that they would not pursue a target incentive if it might be detrimental to the patient.

If their [HbA1c] is 8 , better leave them there because the quality of life is good, instead of giving them high doses and all that, and getting it lower (GP7).

I tend to deal with the problem patients come with first. And then if it's appropriate to ask questions, you know, ticking the boxes, I will do at the end of the consultation (GP10).

There was, however, an interesting tension for a considerable minority of doctors. These physicians described how the generally accepted desire to meet target levels and provide better chronic disease management also meant that at times they felt they might be too proactive in following up patients to meet targets or attend appointments, reflecting a physician-centered, rather than a patient-centered, approach to care.

$\ldots$ it makes it much more likely that we put pressure on those patients to come and have regular checks and to take the treatments that we offer them (GP25).

And in some respects I feel we're less willing to accept them declining treatment (GP52).

I think we're very sort of hung up on figures and numbers and whatever, and not actually looking at what people want or giving them what they want sometimes (PN15).

There was no consensus about whether addressing targeted areas had led to neglecting those areas within the consultation that are not incentivized. Whereas most doctors believed that they treated all patients and patient conditions equally, a minority wondered whether there had been a subtle downgrading of conditions not incentivized in the QOF, as well as the social aspect of family medicine, through changes to available education and increased time pressures within consultations.

I suppose that I, I have less time to attend to social issues and unhappy patients. I tend to try and be sympathetic but within a shorter time period (GP20).

So maybe other data is not sort of recorded quite as well. There's never as much training and education available for 
non-QOF areas as there are for QOF areas, so you don't get the same updating, the same perhaps advice about managing those area (GP1).

\section{Performance Monitoring and Competition}

The interviews highlighted a sense of underlying uncertainty about the future of family medicine in the United Kingdom. Many doctors were worried that there was "no grand plan" within the NHS underpinning the raft of recent reforms. This uncertainty was further aggravated by a negative perception that the new targets formed part of an ever-increasing performance-monitoring and surveillance culture by government agencies.

My honest feeling is that actually nobody has a grand plan, or that there may be several different grand plans which actually don't interrelate with each other, and that people have started initiatives on the basis of one idea and then another initiative on the basis of another idea, and there isn't actually a grand plan (GP49).

There is an environment and ethos of increased surveillance and performance monitoring (GP1).

I suppose it feels more like I'm being watched. It's a little bit like big brother-you've not ticked these boxes (PN2).

There was general agreement that irrespective of the physician's own personal views of the relevance and legitimacy of targets, physicians felt motivated to achieve the highest achievement rate possible for their practice and their own income.

I think that GPs, and doctors by nature, are competitive, and so one wants to get all the brownie points that one can ... (GP20).

The workload has gone up, but then there's more reward if we achieve certain goals, the reward is there. If you don't achieve the reward, you don't get it (GP32).

\section{New Indicators Introduced in 2006}

All doctors and nurses stated that they could see the purpose of most of the indicators in the 2004 iteration of the QOF. More concern was expressed about the relevance to patients of, and work required for achieving, targets introduced in the revised QOF in 2006. These new indicators relate to setting up registers of patients with newly diagnosed chronic kidney disease and obesity and measuring the severity of depression in people with a new diagnosis, which one doctor referred to as "not a family practice way of doing things." No interviewee challenged the importance of these issues or stated their intention not to attempt to meet the targets. A minority of doctors and nurses, however, queried whether these targets were aligned with clear primary health professional objectives and were a core part of family practice and whether they would result in improved patient outcomes.

... the ones where you seem to be collecting data purely for the fact of collecting the data. So I mean initially, for instance, when the renal targets came in and things, it was a case of have a register of people and what stage of chronic kidney disease they were. But there was very little guidance as to what we were supposed to do with them having identified that (GP52).

You know that depression screening ones, because I think someone comes to you, you do a check on them, you immediately, you've known them for a long time and then you think, "Oh, I've got to ask those 2 questions." You get these questions and you feel a bit of a fool because you, you just know by common sense how they are so I don't think that was a great thing to put in (N25).

\section{DISCUSSION}

\section{Strengths and Weaknesses of the Study}

This in-depth qualitative study reports the views of 41 family doctors and nurses working in 22 representative practices across England 3 years after implementation of the pay-for-performance scheme within the new General Medical Services Contract. The study has some limitations. It does not include a patient's perspective, which would have been useful in exploring perceptions of changes in dual consultation agendas. Moreover, the pay-for-performance scheme is evolving, and our study presents a snapshot at a particular point in time. The study did not intend to measure or evaluate actual changes in practice; instead, we relied on physicians' and nurses' accounts of changes. Future research needs to evaluate actual change.

\section{Implications for Practice and Policy}

To obtain active commitment rather than a grudging compliance, ${ }^{4}$ previous research suggests it is important to align quality targets to professional priorities ${ }^{9,22}$ and to ensure the underlying goals of quality targets are understood by those undertaking them. Our findings, however, suggest that these issues are not necessarily relevant when targets are incentivized. Physicians in this study emphasized their commitment to pursuing financially incentivized targets irrespective of whether they agreed with the underlying relevance or goal of the target or whether they thought that targets related to low-yield clinical activities. Almost all physicians in this study had understood the underlying objectives of the indicators included in the original 2004 QOF. ${ }^{1,3}$ There was less enthusiasm, however, for the indicators in the revised 2006 version of the QOF relating to patients with newly diagnosed depression and registers 
of patients with newly diagnosed obesity and chronic kidney disease. These new indicators were perceived as not to be aligned with either a clear objective (eg, a clinical procedure or package of care) or the culture of family medicine (eg, a managing a whole-person rather than simply diagnosing a single condition or risk factor). Despite this lack of enthusiasm, even those who criticized these new indicators were motivated to achieve the targets

Previous research has suggested that financial incentives are effective when they are deemed sufficient to change the behavior necessary to meet the target and when they are used as bonus rewards in addition to existing income. ${ }^{9}$ Professional motivation, while an important component of delivering quality improvement, is not always sufficient on its own. ${ }^{23}$ This nationally representative sample of doctors and nurses believed that the financial incentives had been sufficient to change behavior and to motivate achievement of QOF indicator targets. The QOF was perceived by our study physicians to have increased their income in relation to an increased workload, and the extra income was justified for achieving quality targets rather than as a substitute for existing income. Our findings show, however, that even though achieving the targets required a team effort, the doctors who owned the practice received the target payments themselves, which led to resentment by the other team members (in this case nurses). The study therefore corroborates previous research suggesting that the new contract has had a negative impact on nurses' internal motivation ${ }^{24}$ and may have altered nurse-patient consultations and job satisfaction. ${ }^{25}$

This sample of doctors still believed, as in 2004, that the QOF will have a positive impact on patient health. We found little evidence that nonincentivized conditions were neglected or that doctors' internal motivation was damaged, outcomes feared by the same sample of doctors in 2004. ${ }^{3}$ Our findings confirm, however, some of the predicted unintended consequences of the QOF, including reduced continuity of care and care fragmentation. ${ }^{1,3,11}$ This decline in the opportunity for personal continuity of care is further aggravated by increasing numbers of part-time doctors, which rose from $10 \%$ in 1997 to $25 \%$ in $2007 .{ }^{27}$

Enhancing the role of nurses in the delivery of chronic disease management reinforces the trend in the United Kingdom toward services being provided by a team rather than by an individual physician. Practice nurses have taken on many tasks performed previously by doctors. Although this subspecialization by nurses enables family doctors to focus on assessing and managing poorly differentiated symptoms, ${ }^{26}$ our findings suggest there may be subsequent deskilling of some doctors.
Our respondents reported changes in the context within which services are offered, including the existence of dual patient and QOF agendas in routine 10 -minute consultations. Evidence reported before 2004 suggested that patients found it difficult to voice aspects of their agenda in the consultation, ${ }^{28}$ making patient-centered consultations difficult to achieve. It has also been argued that the QOF has undermined patient-centered care within consultations. ${ }^{29}$ In its current form, the QOF focuses predominately on clinical issues rather than on the personal qualities of physicians and patient-centered care, which have been advocated by some in the United States. ${ }^{6,7}$ We found that the QOF is perceived as changing the clinicians' agenda within consultations by encouraging physicians and nurses to focus on targets in addition to addressing patients' concerns. The concept of 2 (potentially competing) agendas was frequently raised by our study participants. Even though physicians and nurses described how they would give patients' concerns priority, our findings suggest that the QOF might make shared decision making between doctors and patients more difficult to achieve within the limited time constraints of a 10-minute consultation. Despite espousal of support for shared decision making among doctors, ${ }^{30}$ studies indicate that it is an aspiration rather than a reality and that behavioral change is needed by doctors if patients' agendas ${ }^{28}$ are to be heard in the consultation.

In conclusion, a new family medicine pay-for-performance scheme in the United Kingdom may have achieved its declared objectives of improving diseasespecific processes of patient care through reaching a series of specific evidence-based targets. Our findings, however, suggest that QOF has changed the way doctors and nurses work together and the nature of clinician-patient consultations. While acknowledging the positive impact of the QOF on patient outcomes and physician income, many physicians expressed concerns about the future role of UK family practitioners, as well as worries about the consequences of a performance-monitoring culture. Further research is planned to observe the evolution and multiple effects of this dynamic pay-for-performance scheme.

To read or post commentaries in response to this article, see it online at http://www.annfammed.org/cgi/content/full/6/3/228.

Submitted November 1, 2007; submitted, revised, February 14, 2008; accepted February 16, 2008.

Key words: Pay-for-performance; primary health care; United Kingdom

Acknowledgments: The authors wish to thank the physicians and nurses who agreed to be interviewed. The authors also wish to thank Nan Bailey, Angela Swallow, Ella Gaehl, and Nicola Small. 


\section{References}

1. Roland M. Linking physician pay to quality of care: a major experiment in the UK. N Engl J Med. 2004;351(14):1448-1454.

2. British Medical Association. Revisions to the GMS contract, 2006/07. Chapter 2. Improving quality in the UK. http://www.bma. org.uk/ap.nsf/Content/revisionnGMSFeb20062 chap2impquality. Accessed Oct 29, 2007.

3. Roland MO, Campbell SM, Bailey N, Whalley D, Sibbald B. Financial incentives to improve the quality of primary care in the UK: predicting the consequences of change. Prim Care Res Dev. 2006; 7:18-26.

4. Fisher ES. Paying for performance-risks and recommendations. N Engl J Med. 2006;355(18):1845-1847.

5. Young GJ, White B, Burgess JF, et al. Conceptual issues in the design and implementation of pay for quality programs. A J Med Quality. 2005;20(3):144-150.

6. Rowe JW. Pay for performance and accountability: related themes in improving health care. Ann Intern Med. 2006;145(9):695-699.

7. Safavi K. Patient centred pay for performance: are we missing the target? J Healthc Manag. 2006;51(4):215-218.

8. Town $R$, Kane $R$, Johnson $P$, Butler $M$. Economic incentives and physicians' delivery of preventive care: a systematic review. Am I Prev Med. 2005;28(2):234-240

9. Bokhour BG, Burgess JF, Hook JM, et al. Incentive implementation in physician practices: a qualitative study of practice executives perspectives on pay for performance. Med Care Res Rev. 2006;63 (1 Suppl):73S-95S.

10. Binderman J, Kilo CM, Oldham J. Rethinking incentives. J Med Pract Manage. 2000;16(2):70-74.

11. Petersen LA, Woodard LD, Urech T, Daw C, Sookanan S. Does pay for performance improve the quality of care? Ann Intern Med. 2006;145(4):265-272.

12. Wright J, Martin D, Cockings S, Polack C. Overall quality and outcomes framework scores lower in deprived areas. Br J Gen Pract. 2006:56(525):277-279.

13. Ashworth M, Seed P, Armstrong D, Durbaba S, Jones R. The relationship between social deprivation and the quality of care: a national survey using indicators from the quality and outcomes framework. Br J Gen Pract. 2007;57(539):441-448.

14. Doran T, Fullwood C, Gravelle H, et al. Family practice performance in the first year of the UK's new pay for performance scheme: good clinical practice or 'gaming'? N Engl J Med. 2006;355(4):375-384.
15. Guthrie B, McLean G, Sutton M. Workload and reward in the Quality and Outcomes Framework of the 2004 general practice contract. Br J Gen Pract. 2006;56(532):836-841.

16. Sutton M, McLean G. Determinants of primary care medical quality measured under the new UK contract: cross sectional study. BMJ. 2006;332(7538):389-390.

17. Tahrani AA, McCarthy M, Godson J, et al. Diabetes care and the new GMS contract. Br J Gen Pract. 2007;57(539):483-485.

18. Campbell S, Reeves D, Kontopantelis E, Middleton E, Sibbald B Roland M. Quality of primary care in England with the introduction of pay for performance. N Engl J Med. 2007;357(2):181-190.

19. Campbell SM, Roland MO, Middleton E, Reeves D. Improvements in the quality of clinical care in English general practice 1998-2003: longitudinal observational study. BMJ. 2005;331(7525):1121-1125.

20. Strauss A, Corbin J. Basics of Qualitative Research: Grounded Theory Procedures and Techniques. London: Sage Publications; 1990.

21. Silverman D. Interpreting Qualitative Data. Methods for Analysing Talk, Text and Interaction. London: Sage Publications; 1997.

22. Spooner A, Chapple A, Roland M. What makes British general prac titioners take part in a quality improvement scheme? J Health Serv Res Policy. 2001;6(3):145-150.

23. Institute of Medicine. Committee on Quality of Health Care in America. Crossing the Quality Chasm: A New Health System for the 21st Century. Washington DC. National Academy Press; 2001.

24. McDonald R, Harrison S, Checkland K, Campbell SM. Roland M. Impact of financial incentives on clinical autonomy and internal motivation in primary care: ethnographic study. BMJ. 2007;334(7608):1333-1334

25. Mercer SW, McGregor W. New contract reduces quality of patientnurse relationship. BMJ. 2007;334(7583):8.

26. Wilson T, Roland MO, Ham C. The contribution of general practice and the general practitioner to NHS patients. J R Soc Med. 2006;99(1):24-48

27. Information Centre. General and Personal Medical Services England 1996-2006. The Information Centre, London; 2007

28. Barry CA, Bradley CP, Britten N, Stevenson FA, Barber N. Patients' unvoiced agendas in general practice consultations: qualitative study. BMJ. 2000;320(7244):1246-1250.

29. Mangin D, Toop L. The quality and outcomes framework: what have you done to yourselves? Br J Gen Pract. 2007;57(539):570-572.

30. Elwyn G, Edwards A, Kinnersley P, Grol R. Shared decision making and the concept of equipoise: the competencies of involving patients in healthcare choices. Br J Gen Pract. 2000;50(460):892-897. 